\title{
Physicians indicated the need to frame questions and develop indirect approaches that foster patient trust in evaluating victims of domestic violence
}

Gerbert B, Caspers N, Bronstone A, et al. A qualitative analysis of how physicians with expertise in domestic violence approach the identification of victims. Ann Intern Med 1999 Oct 19;131:578-84.

QUESTION: How do physicians identify victims of domestic violence in healthcare encounters?

Design

Focus groups.

\section{Setting}

Research facilities in the San Francisco Bay area, USA.

\section{Participants}

45 emergency department, obstetrician/gynaecologist, and primary care physicians (mean age 43 y, $53 \%$ women) who worked with patients $\geqslant 20$ hours per week, and identified and intervened with victims of domestic violence.

\section{Methods}

2 moderators facilitated 6 focus groups, each with $6-11$ physicians of the same specialty. The groups met for 90 minutes in a focus group setting. A semi-structured guide was used which allowed the moderators to follow certain topics and open new lines of inquiry. Audiotapes of the focus group sessions were transcribed and the study investigators conducted multiple readings of the transcripts to identify prominent themes. Results were directed back to 4 research participants to determine if the experiences of their focus group were reflected.

\section{Main results}

5 major themes emerged that revealed how physicians from any of the specialties evaluated victims of domestic violence. The first theme, how physicians framed screening questions to reduce patient discomfort and fear, described how physicians used standardised screening questions that were carefully framed to minimise patient discomfort and fear of being identified.

The second theme, patient signs and symptoms that "switched on a light bulb" to suspect domestic violence, emphasised the need to pursue the identification of domestic violence when patients presented with certain constellations of symptoms, injuries, or signs that "switched on a light bulb in their heads".

The third theme, direct and indirect approaches to identification with an emphasis on facilitating patient trust and disclosure over time, revealed that the more time physicians had, the more indirectly they approached the topic, and that developing a trusting relationship over time increased the chance of future disclosure.

The fourth theme, the rarity of direct patient disclosure, revealed that direct patient disclosure occurred rarely and only when the patient had an acute injury.

The fifth theme, how they redefined successful outcomes of universal screening, relates to the need for a redefinition of successful outcomes so that compassionate asking, in and of itself, rather than gaining direct disclosure, constitutes success.

Time-squeezed physicians also described 2 barriers to screening for domestic violence: (1) mandatory reporting, and (2) burnout because of lack of direct disclosure.

\section{Conclusion}

Physician reports showed the need to frame questions and develop indirect approaches that foster patient trust when evaluating and treating victims of domestic violence.

\section{COMMENTARY}

This study by Gerbert et al identifies a primary medical care standard for physicians working with groups at risk of domestic violence. Their results support the importance of having the time for indirect approaches to improving doctor-patient communication by fostering patient trust. These findings are consistent with the clinical studies published in psychiatry and forensic psychiatry which indicate that the building of a therapeutic alliance, by helping patients develop trust, analysis, and communication skills, is essential in effective prediction and prevention of domestic violence. ${ }^{1}$ As such, we can now speak with reasonable confidence about unity of medical and mental standards for domestic violence prevention across the clinical specialty spectrum.

The importance of primary care physicians having the time to show compassionate concern toward potential victims of domestic violence is highlighted. Thus, the time-squeezed primary physician who simply makes a "blind" referral to a mental health professional of a potential victim or even a potential perpetrator of domestic violence, may well be acting counter therapeutically-in effect, abandoning a patient. There already are relevant American Medical Association ethics codes warning against inappropriate termination and patient abandonment. ${ }^{2}$ Primary care physician involvement is often key to violence prevention.

Both mandated reporting and managed care driven requests for documentation may inhibit some potential victims from reporting. By the same token, the relative immunity from civil action currently enjoyed by some managed care organisations has promoted practices which lead physicians to be time-squeezed with impunity. When clinicians are mindful of potential pressures and conflicts of interest, however, they can still act effectively to prevent domestic violence through enhancing doctor-patient-victim system communication and engaging in an informed

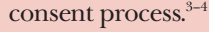

\section{Harold J Bursztajn, MD Harvard Medical School Cambridge, Massachusetts, USA}

1 Gutheil TG, Bursztajn H, Brodsky A. The multidimensional assessment of dangerousness: competence assessment in patient care and liability prevention. Bull Am Acad Psychiatry Law 1986;14:123-9.

2 Code of Medical Ethics: Current Opinions with Annotations, 1998-1999. 8.115 and 8.132. Chicago, IL: AMA, $1996 ; 125,128$.

3 Wulsin LR, Bursztajn H, Gutheil TG. Unexpected clinical features of the Tarasoff decision: the therapeutic alliance and the "duty to warn". Am J Psychiatry 1983;140:601-3.

4 Bursztajn HJ, Brodsky A. Captive patients, captive doctors: clinical dilemmas and interventions in caring for patients in managed health care. Gen Hosp Psychiatry 1999;21:239-48.
Source of funding: National Institute of Mental Health

For correspondence: $D$ $B$ Gerbert, Division of Behavioral Sciences, University of California, San Francisco, 601 Montgomery Street, Suite 810, San Francisco, CA 94111 USA. Fax +1415502 7314. 